\title{
Does arousal frequency predict daytime function?
}

\author{
R.N. Kingshott*, H.M. Engleman*, I.J. Deary , N.J. Douglas*
}

Does arousal frequency predict daytime function? R.N. Kingshott, H.M. Engleman, I.J. Deary, N.J. Douglas. CERS Journal Ltd 1998.

ABSTRACT: Patients with the sleep apnoea/hypopnoea syndrome (SAHS) have impaired daytime function with demonstrable sleepiness and impaired cognition. The hypothesis that brief arousals from sleep cause these daytime impairments was tested.

One hundred and fifty patients with sleep disordered breathing were studied prospectively, comparing overnight polysomnography with daytime measures of objective sleepiness, psychological well-being and cognitive performance.

Significant, but weak $\left(r^{2}<0.1\right)$, relationships were seen between several nocturnal measures (apnoea/hypopnoea index, arousals and desaturation variables) and daytime measures of quality of life, well-being, subjective sleepiness, symptoms and cognitive performance. The only significant relationship between nocturnal variables and objective sleepiness was a very weak correlation $\left(r^{2}<0.05\right)$ between the lowest oxygen saturation and mean maintenance of wakefulness test (MWT) result. The MWT was better correlated with daytime function than the multiple sleep latency test.

This study shows a lack of strong relationships between conventional nocturnal measures and daytime function in patients with sleep disordered breathing. Eur Respir J 1998; 12: 1264-1270.

Patients with the sleep apnoea/hypopnoea syndrome (SAHS) suffer from excessive daytime sleepiness [1, 2], cognitive decrements $[3,4]$ and decreased psychological well-being [5]. These daytime effects lead to an increased risk of vehicle accidents [6], work inefficiency [7] and poor social relationships [7]. Previous correlational studies [3, $5,8,9]$ have looked at possible causative relationships between the nocturnal features of SAHS and daytime function. However, there is still controversy as to what extent these daytime deficits are due to sleep fragmentation, caused by brief microarousals from sleep [9], or to intermittent nocturnal hypoxaemia associated with the sleep disordered breathing [4]. Studies by Gulleminault et al. [8] and RoEHRS et al. [9] found measures of sleep disturbance and sleep fragmentation, respectively, to be the best predictors of excessive daytime sleepiness. In contrast, BEDARD et al. [4] found hypoxaemia best predicted daytime sleepiness and CHESHIRE et al. [5] found no nocturnal correlates of daytime sleepiness, but did find that hypoxaemia and sleep fragmentation significantly correlated with measures of cognitive function.

These conflicting results have tended to be based on older definitions of arousal [1, 4, 8, 9] and/or relatively small numbers of subjects $[1,4,5]$. It has recently been shown that brief arousals from sleep produce sleepiness [10-12] and impair daytime function [10]. The primary aim of the current study was to investigate the hypothesis that brief arousals from sleep cause the sleepiness and impaired daytime function found in SAHS. The secondary aim of this study was to determine whether the multiple sleep latency test (MSLT) [13] or maintenance of wakefulness test (MWT) [14] is better related to daytime function in SAHS patients.

For editorial comments see page 1239.
*Respiratory Medicine Unit and $\ddagger$ Dept of Psychology, University of Edinburgh, UK.

Correspondence: N.J. Douglas

Respiratory Medicine Unit

Dept of Medicine

University of Edinburgh

Royal Infirmary

Edinburgh

EH3 9YW

UK

Fax: 441315363255

Keywords: Cognitive function sleep apnoea/hypopnoea syndrome sleepiness

Received: April 231998

Accepted after revision September 151998

Methods

Subjects

A prospective study on consecutive patients clinically referred to the sleep centre with possible SAHS was performed. All study patients had either self-reported sleepiness (defined as Epworth sleepiness scale (ESS)Š8) or two other major symptoms of SAHS [2] (table 1). Patients were aged between 18 and 75 yrs old and living within 50 miles of the sleep centre. Patients who had coexisting causes of daytime sleepiness (e.g. night or rotating shiftworkers; self-reported average sleep duration $<5 \mathrm{~h}$ ), major psychiatric and neurological disorders, and coexisting causes of hypoxaemia were excluded. Following scoring of the diagnostic polysomnography, patients with an apnoea/hypopnoea index $(\mathrm{AHI})<5$ or with evidence of coexisting sleep disorders were also excluded. One hundred and sixty-seven consecutive patients were eligible for the study and 150

Table 1. - Percentages of self-reported patient symptoms $(\mathrm{n}=150) *$

\begin{tabular}{lc}
\hline Self-reported symptom & Patients reporting symptoms \\
& $\%$ \\
\hline Snoring & 100 \\
Unrefreshing sleep & 77 \\
Daytime sleepiness (ESS Šs) & 75 \\
Witnessed apnoeas & 49 \\
Choking episodes & 33 \\
Sleepiness whilst driving & 21 \\
Nocturia & 9 \\
*: all symptoms reported from the authors in-house symptom
\end{tabular}
questionnaire; ESS: Epworth sleepiness scale. 
Table 2. - Patients population data $(n=150)$

\begin{tabular}{|c|c|c|}
\hline Measurement & Mean (SD) & Range \\
\hline Age yrs & $50(10)$ & $22-71$ \\
\hline $\mathrm{BMI} \mathrm{kg} \cdot \mathrm{m}^{-2}$ & $32(7)$ & $20-64$ \\
\hline AHI events $\cdot h$ slept $^{-1}$ & $45(31)$ & $7-144$ \\
\hline Arousal index arousal.h slept ${ }^{-1}$ & $51(28)$ & $8-141$ \\
\hline Minimum oxygen saturation $\%$ & $83(11)$ & 34-95 \\
\hline$\%$ Wake* & $22(12)$ & $2-61$ \\
\hline$\%$ Stage 1 sleep* & $8(7)$ & $0.3-35$ \\
\hline$\%$ Stage 2 sleep* & $44(11)$ & $15-72$ \\
\hline$\% \mathrm{SWS} *$ & $11(7)$ & $0-38$ \\
\hline$\%$ REM sleep $*$ & $15(6)$ & $1.6-30$ \\
\hline Epworth sleepiness scale & $12(5)$ & $0-24$ \\
\hline MSLT-mean SOL min & $9.7(4.7)$ & $2.5-20$ \\
\hline MWT-mean SOL min & $27(12)$ & $2.6-40$ \\
\hline
\end{tabular}

*: values for sleep stages are expressed as a percentage of sleep period time. BMI: body mass index; AHI: apnoea/hypopnoea index; SWS: slow wave sleep; REM: rapid eye movement; MSLT: multiple sleep latency test; MWT: maintenance of wakefulness test; SOL: sleep onset latency.

(136 males; table 2) agreed to participate. Seventeen declined because of work pressures $(n=14)$, family commitments $(\mathrm{n}=1)$ and a poor night's sleep $(<6 \mathrm{~h}$, subjective estimation) prior to daytime testing $(\mathrm{n}=2)$.

\section{Nocturnal measurements}

All patients underwent full nocturnal polysomnography according to the authors' standard methods [15]. Sleep was monitored by electroencephalography (EEG) (CZ-PZ), electro-oculography (EOG) and submental electromyography (EMG). Thoracic and abdominal respiratory movements were measured by inductance plethysmography, oronasal airflow using thermocouples and arterial oxygen saturation using pulse oximetry (Ohmeda Biox 3700; Louisville, CO, USA). Electrocardiogram (ECG), microphone snoring sounds and right and left leg movements were also monitored. All signals were recorded onto a computerized system (Compumedics S, Abbotsford, Victoria, Australia) using a 16-channel polygraph configuration.

\section{Off-line analysis}

Sleep stages were manually scored using standard RеснтSCHAFFEN and KALES [16] (R\&K) scoring guidelines. An apnoea was defined as a complete cessation of airflow for a minimum of $10 \mathrm{~s}$, and a hypopnoea as a $50 \%$ reduction in thoracoabdominal movement for a minimum of $10 \mathrm{~s}$ [17]. The total number of respiratory events was divided by total sleep time (TST) to give an AHI. Microarousals were scored using the Cheshire definition [5, 18] of a return to theta or alpha on the EEG for $>1.5 \mathrm{~s}$, with a concurrent rise in EMG tone, however brief. Both spontaneous and respiratory event-related arousals were included in the microarousal index. Oxygen desaturations of $\breve{S} 2,3$ and $4 \%$ of baseline were calculated from the overnight study using automatic desaturation detection (Compumedics S) and divided by TST to give a desaturation index. In addition, the awake saturation and the minimum saturation were measured directly from the oximetry channel.

\section{Daytime assessments}

All patients underwent a battery of daytime tests to measure a wide variety of functions affected by SAHS [5, 19] (table 3). All tests were applied at the same time of day in all subjects, after a normal night's sleep at home within one month of their overnight polysomnography. Objective daytime sleepiness was measured using both the MSLT [13] and MWT [14]. All naps were terminated after two consecutive epochs of RECHTSCHAFEN and KaLES [16] sleep, or if no sleep occurred, after $20 \mathrm{~min}$ on the MSLT, and $40 \mathrm{~min}$ on the MWT. The sleep onset latency (SOL) was determined from "lights out" until the first 30-s epoch of any stage of sleep [26]. The mean SOL was calculated from the average of the four MSLT naps and MWT naps, respectively, across the day. Questionnaires were administered in the morning after the 10:00 h MSLT (table 3), and included self-ratings of symptoms, sleepiness and well-being as previously described [10, 19, 27], with the addition of the Short Form (SF) 36 questionnaire [23], measuring quality of life. A battery of cognitive function tests were given in the afternoon of the daytime session as previously described [10, 19], with the addition of a 10-min computerized simple unprepared response time (SURT) task, measuring information processing time and attention (table 3). Patients were instructed to withdraw from caffeine on the evening prior to the daytime testing, and throughout the test day decaffeinated drinks were provided.

\section{Table 3. - Timetable of daytime assessments}

09:00h Wire up patient, anthropometry (height, weight, collar and waist sizes), background questionnaire. (Minimum 20 min free time.)

10:00h MSLT

Questionnaires (Stanford sleepiness scale (SSS), Epworth sleepiness scale (ESS) [20], Hospital anxiety and depression (HAD) scale [21], Nottingham health profile (NHP) part 2 [22], Short Form (SF) 36 [23], SAHS symptom questionnaire). (Minimum 20 min free time.)

11:00h MWT

Finish any remaining questionnaires. (Minimum 15 min free time.)

12:00h MSLT

Lunch

(Minimum $40 \mathrm{~min}$ free time.)

13:00h MWT

Simple unprepared reaction time (SURT) task. (Minimum 10 min free time.)

14:00h MSLT

Cognitive function battery: Paced Auditory Serial Addition Test (PASAT) [24], Digit symbol substitution [25], Block Design [25] (WAIS-R subtests), Trail Making A and $\mathrm{B}[25]$.

(Minimum 10 min free time.)

15:00h MWT

(Minimum 20 min free time.)

16:00h MSLT

Steer clear for $30 \mathrm{~min}$.

(Minimum 10 min free time.)

17:00h MWT

MSLT: multiple sleep latency test; SAHS: sleep apnoea/hypopnoea syndrome; MWT: maintenance of wakefulness test; WAIS$\mathrm{R}$ : revised Wechsler adult intelligence scale. 


\section{Statistical analysis}

Relationships between nocturnal variables and daytime function and relationships between daytime outcome measures were evaluated by Spearman rank correlations. All correlations were partial, controlling for age and awake oxygen saturation. Multiple regression analysis was used to identify independent predictors when an outcome measure significantly correlated with more than one variable. As age [25] and awake oxygen saturation [28] affect daytime function, they were forced into the multiple regression analysis prior to stepwise regression of the other significant correlates. All tests were two-tailed and $\mathrm{p}<0.05$ was accepted as statistically significant. All data were analysed using SPSS for Windows 6.1. (SPSS Inc, Chicago, IL, USA). For reasons of brevity, only daytime variables which correlated significantly with at least one polysomnography variable are shown in the tables.

\section{Results}

\section{Nocturnal correlates of daytime function}

Objective daytime sleepiness. Neither arousal index nor AHI significantly correlated with objective sleepiness, as measured by the MSLT or MWT (fig. 1, table 4). Only minimum oxygen saturation correlated significantly, but weakly, with MWT (table 4).

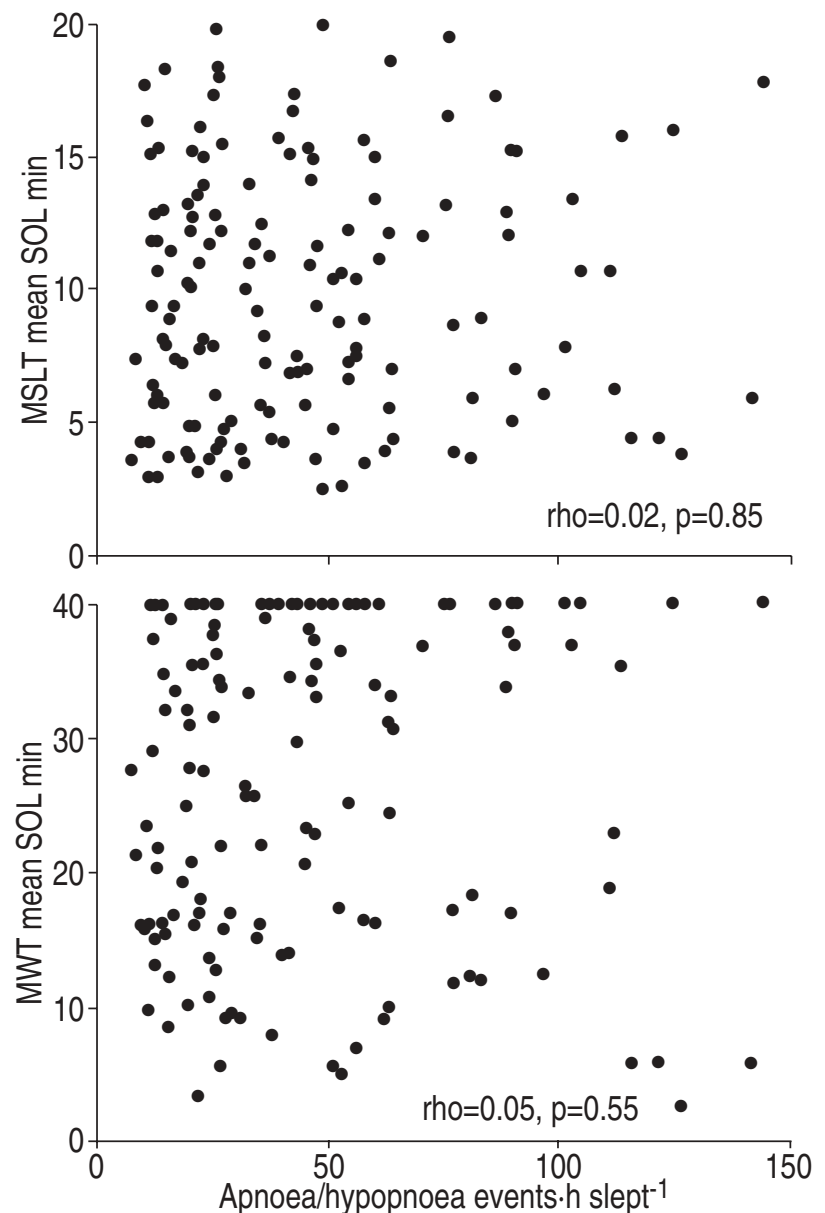

Self-ratings of daytime function. Significant, but weak, relationships were also found between arousal index, AHI and desaturation variables, and measures of daytime function (table 4). Multiple regression analysis found the arousal index to be the single independent predictor of the SF36 physical components summary score accounting for $7 \%$ of the variance. A $2 \%$ desaturation index was the single independent predictor for the SF36 health transition (10\% of variance) and general health ( $8 \%$ of variance) scores. A 3\% desaturation index predicted SF36 physical function score accounting for $10 \%$ of the variance, and minimum saturation predicted scores for SF36 vitality (12\% of variance), Nottingham Health Profile ( $8 \%$ of variance) and the SAHS symptom questionnaire ( $9 \%$ of variance).

Cognitive performance. Arousal index significantly correlated with reaction time gaps $(1 \mathrm{gap}=$ reaction time response $>1$ s) and AHI with performance intelligence quotient (IQ). Measures of hypoxaemia also significantly correlated with reaction time gaps and performance IQ, along with Trail Making B, Paced Auditory Serial Addition Test (PASAT) and Digit symbol scores (table 4).

\section{Daytime interrelationships}

MSLT versus $M W T$. There was a statistically significant relationship between the MSLT and MWT ( $\mathrm{r}=0.43, \mathrm{p}<$

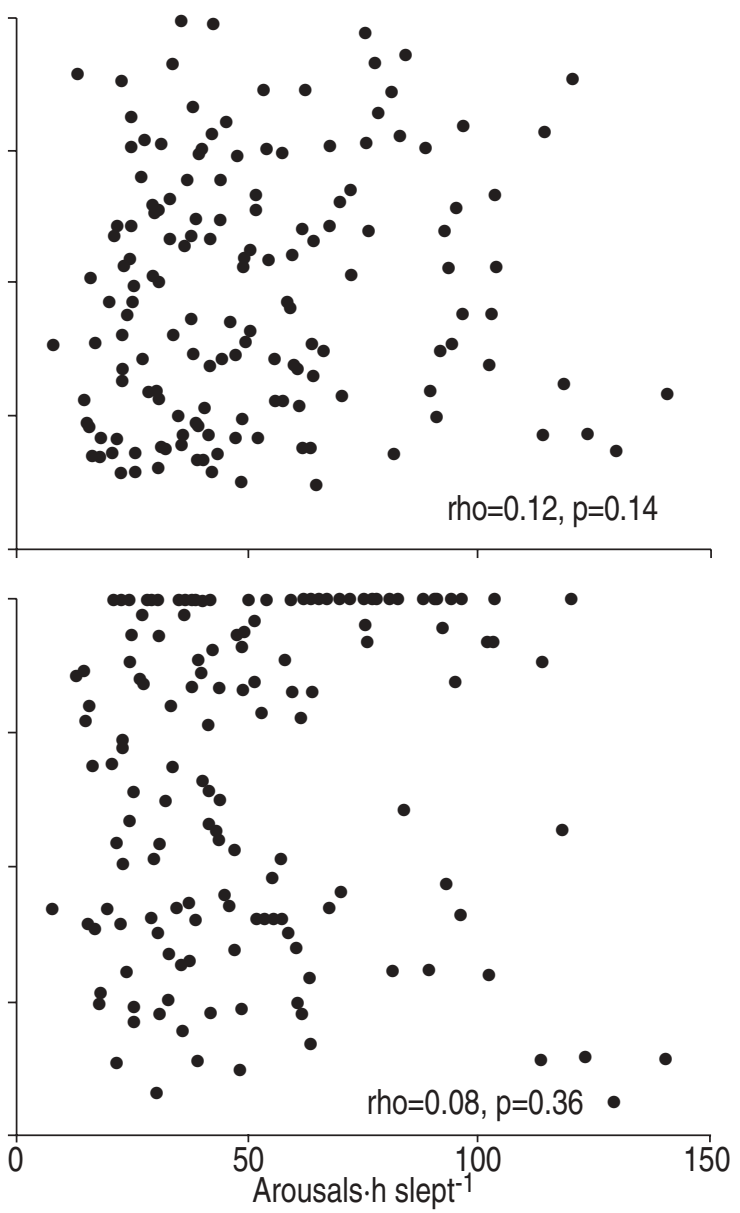

Fig. 1. - Nonsignificant relationships between sleep variables and daytime sleepiness. MSLT: multiple sleep latency test; SOL: sleep onset latency; MWT: maintenance of wakefulness test. 
Table 4. - Spearman rank correlation matrix showing relationships between nocturnal and daytime function variables $^{+}$

\begin{tabular}{|c|c|c|c|c|c|c|}
\hline $\begin{array}{l}\text { Daytime } \\
\text { function } \\
\text { measures }\end{array}$ & ArI & AHI & $2 \%$ & $3 \%$ & $4 \%$ & Min sat \\
\hline Mean MSLT & 0.12 & 0.02 & 0.12 & 0.07 & 0.07 & -0.06 \\
\hline Mean MWT & 0.08 & 0.05 & 0.03 & -0.30 & -0.03 & $0.19 *$ \\
\hline \multicolumn{7}{|l|}{ SF36: } \\
\hline PCS & $-0.22 *$ & $-0.20 *$ & $-0.20 *$ & $-0.23 *$ & $-0.23 * *$ & $* 0.21 *$ \\
\hline General health & $-0.24 * *$ & $-0.22 *$ & $-0.29 * *$ & $-0.28 *$ & $-0.27 * *$ & $0.25 * *$ \\
\hline Heal & $0.20 *$ & $0.24 * *$ & $0.29 *$ & $0.28 *$ & $0.28 * *$ & $*_{-}-0.25 * *$ \\
\hline al func. & -0.09 & -0.08 & $-0.18 *$ & $-0.23 *$ & $-0.22 * *$ & $* 0.22 *$ \\
\hline Social func. & -0.01 & -0.05 & -0.09 & -0.11 & -0.10 & $0.17 *$ \\
\hline Vitality & -0.05 & -0.16 & $-0.22 *$ & $-0.25 *$ & $-0.25 * *$ & $* 0.32 * * *$ \\
\hline ESS & 0.03 & -0.04 & 0.06 & 0.07 & 0.06 & $-0.22 *$ \\
\hline NHP & -0.02 & 0.05 & 0.16 & $0.19 *$ & $0.19 *$ & $-0.26 * *$ \\
\hline Symptom score & 0.10 & 0.16 & $0.23 * *$ & $0.27 *$ & $0.27 * *$ & $*_{-}-0.33 * * *$ \\
\hline \multicolumn{7}{|l|}{ HAD depression } \\
\hline score & -0.05 & -0.04 & 0.08 & 0.11 & 0.12 & $-0.21 *$ \\
\hline DSS & -0.07 & -0.12 & $-0.18 *$ & -0.15 & -0.12 & 0.11 \\
\hline PAS & -0.08 & -0.12 & $-0.17 *$ & -0.14 & -0.13 & 0.15 \\
\hline B & 0.07 & 0.06 & 0.13 & 0.15 & 0.16 & $-0.17 *$ \\
\hline Performance IQ & -0.10 & $-0.17 *$ & $-0.23 *$ & $-0.22 *$ & $-0.19 *$ & $0.19 *$ \\
\hline on time & & & & & & \\
\hline & $0.19 *$ & 0.12 & 0.13 & 0.14 & 0.12 & $-0.17^{*}$ \\
\hline
\end{tabular}

+: all correlations are controlled for age and awake oxygen saturation; *: $\mathrm{p}<0.05 ; * *: \mathrm{p}<0.01 ; * * *$ : $\mathrm{p}<0.001$. ArI: arousal index; AHI: apnoea/hypopnoea index; $2-4 \%$ : $2-4 \%$ oxygen desaturation; Min sat: minimum oxygen saturation; MSLT: multiple sleep latency test; MWT: maintenance of wakefulness test; PCS: physical components summary; SF36: short form 36; trans.: transition; func.: functioning; ESS: Epworth sleepiness scale; NHP: Nottingham health profile; HAD: hospital anxiety and depression scale; DSS: digit symbol substitution; PASAT: Paced Auditory Serial Addition Test; IQ: intelligence quotient.

0.0001). Ceiling effects were seen on the MWT, with 40 patients $(27 \%)$ not sleeping on any of the four MWT trials, whilst only one patient $(0.7 \%)$ failed to sleep on any of the four MSLT naps.

Objective sleepiness versus self-ratings of daytime function. The MWT significantly correlated with a range of self-reports of daytime function in the expected direction. The MSLT only significantly correlated weakly with the ESS (table 5).

Objective sleepiness versus cognitive performance. The MWT significantly correlated with a wide range of performance tasks in the expected direction. No such relationships were seen between the MSLT and cognitive measures (table 5).

\section{Discussion}

This study shows that conventional variables scored from routine polysomnography do not closely relate to impairments in daytime function found in patients with the sleep apnoea/hypopnoea syndrome.

A variety of nocturnal measures were significantly correlated with daytime function, but in no case explained more than $12 \%$ of the observed variance. There was a tendency for saturation measures to correlate better with day-
Table 5. - Spearman rank correction matrix showing relationships between measures of daytime function ${ }^{+}$

\begin{tabular}{lll}
\hline Daytime function measures & MSLT & MWT \\
\hline Epworth sleepiness scale & $-0.23^{* *}$ & $-0.48^{* * *}$ \\
Nottingham health profile part 2 & -0.10 & $-0.31^{* * *}$ \\
SF36 Health transition & -0.08 & $-0.28^{* *}$ \\
SF36 Physical function & -0.005 & $0.20^{*}$ \\
SF36 Social function & 0.07 & $0.28^{*}$ \\
SF36 Physical component summary & 0.02 & $0.23^{* *}$ \\
HAD Depression & -0.03 & $-0.22^{*}$ \\
SAHS symptom score & -0.04 & $-0.22^{*}$ \\
Steer clear \% cow hits & -0.13 & $-0.30^{* * *}$ \\
PASAT (4 s) & -0.003 & $0.17^{*}$ \\
Mean reaction time & -0.15 & $-0.32^{* * *}$ \\
Block design & -0.01 & $0.20^{*}$ \\
Performance IQ & -0.10 & $0.22^{* *}$ \\
\hline
\end{tabular}

$+:$ all corrections are controlled for age and awake oxygen saturation; *: $\mathrm{p}<0.05 ; * *: \mathrm{p}<0.01 ; * * *: \mathrm{p}<0.001$. MSLT: multiple sleep latency test; MWT: maintenance of wakefulness test; SF36: short form 36; HAD: hospital anxiety and depression scale; SAHS: sleep apnoea/hypopnoea syndrome; PASAT: Paced Auditory Serial Addition Task; IQ: intelligence quotient.

time function than arousals or AHI. SMITH and SHNEERSON [23] looked at ratings on the SF36 questionnaire in snorers and SAHS patients and found $4 \%$ desaturations weakly correlated with vitality, general health and physical function, in agreement with the current study. Those authors speculated that if sleep fragmentation had been measured, stronger relationships may have been seen, but this was not found in the current study.

This study confirms the authors' previous observation [29] that there is no significant relationship between ESS and AHI or arousal frequency. This contrasts with a study by JoHNS [20], where relationships were found between ESS and AHI $(r=0.4, p<0.001)$ in SAHS patients. In agreement with earlier studies [3-5], AHI, arousal index and especially measures of hypoxaemia were found in the present study to weakly predict deficits in cognitive performance, in particular sustained attention, mental flexibility, visuomotor co-ordination and processing time. Unlike previous studies on cognitive function in SAHS this study controlled for age [3, 4] and awake saturation [5] and used a much larger sample size, and included all patients with an AHI Š5. The results are compatible with a recent population study showing significant, but weak, correlations between AHI and neuropsychological deficits [30].

Neither the brief microarousals nor respiratory events correlate with measures of excessive daytime sleepiness in patients with sleep disordered breathing, in agreement with a previous small study [5]. However, other larger studies have found weak $(r<0.37)$ relationships with objective daytime sleepiness [9, 14]. RoEHRS et al. [9] found multiple significant, but weak, relationships between nocturnal variables and daytime sleepiness as measured by the MSLT. Multiple regression analysis found respiratory related arousals (defined as a $\breve{S} 3$ s increase in EEG and/or EMG frequency at the termination of a respiratory event) to be the single independent predictor of daytime sleepiness. Similar findings were presented by POCETA et al. [14] using the MWT to measure daytime sleepiness. However, in both of these studies, although relationships are statistically significant, only $13 \%$ of the variance in daytime sleepiness is 
explained by their measures of sleep fragmentation. The present study thus confirms and extends these studies by indicating that there is no close relationship between either newer microarousal definitions or other nocturnal measures and objective sleepiness. One significant relationship, between minimum oxygen saturation and MWT, was found, when controlling for awake oxygen levels and age. This was a weak relationship and could be a type 1 statistical error given the multiple comparisons. However, BÉDARD et al. [4] also found a significant but weak relationship between MSLT and minimum oxygen saturation.

\section{Daytime interrelationships}

In agreement with SANGAL et al. [31], a significant relationship between the MSLT and MWT was found in the present study, but with considerable discordance between these two tests. Some patients have a high MSLT latency but low MWT latency, which confirms that they are measuring different physiological abilities. It was also found that the MWT correlated much more closely than the MSLT with measures of quality of life, well-being, symptoms and sleepiness ratings and with a diverse range of cognitive function tests measuring general functioning, reaction time and concentration. The MSLT was significantly related to the ESS alone. The data thus suggest that the MWT better reflects daytime function in patients with sleep-disordered breathing than the MSLT.

\section{Potential criticisms}

One of the potential criticisms of this study is the large number of correlations performed. Indeed, 228 correlations were sought. Given this, it is inevitable that some of the 61 significant correlations observed are due to chance alone, but only 11 such chance observations would be expected $(228 / 20)$ given the significance value set at $\mathrm{p}<0.05$. However, the main message of this study is not the number of significant correlations but the weakness of the correlations observed. In fact, of the correlations which examined the primary hypothesis that arousals would predict function, only 4 of 29 were significant, all explaining $<2 \%$ of the variance.

Another possible criticism of this study is the use of the authors' own validated definition of arousal $[5,18]$ which could be a cause of the weakness of the relationships with arousals found in the study. However, a recent study by COLlaRd et al. [32] found significant relationships between the movement arousal index and the magnitude of sleep quality and disordered breathing, and yet a "fairly similar" [32] arousal definition was used but only minimal relationships with daytime function outcomes were found. In addition the arousal definition used in the present study gives results closely similar to those of the standard American Sleep Disorder Association (ASDA) definition [18]. As well as using this 1.5-s microarousal definition, the possibility of whether a better fit existed between a measure of more sustained awakening (RECHTSCHAFFEN and KALES [16] awakenings) and daytime function variables was also examined. There was not.

Nocturnal polysomnography measures vary in their signal and scoring reliability. Desaturation measures are reproducible and indices are computer derived, whereas arousals and AHI have lower, although still good [18, 33], reproducibility and are visually scored. These differences may contribute to why desaturation indices were more closely related to daytime function.

Potential problems also exist in using the MSLT and MWT as "gold standards" for sleepiness. Both tests can be affected by environmental and behavioural influences. The MSLT SOL can be reduced by a financial incentive if a subject "falls asleep faster than the last time", compared to a nonincentive group [34]. The MWT is highly susceptible to motivational factors, and instructing an individual to "try and stay awake" may activate a whole range of physiological processes such as motivation, competitiveness [35] and attention ability [14] which are likely to vary between individuals. There may be a role for testing other measures of daytime sleepiness, including behavioural tasks [36].

Patients who were sleepy in terms of their ESS score were included as the authors did not want to exclude those sleepy patients who had lost their frame of reference and therefore underestimate their sleepiness [37]. Seventy-five per cent of the patients had ESS S8 and a further $2 \%$ had ESS $<8$ but admitted having problems with sleepiness whilst driving. All the remainder had at least two major features of SAHS and an AHI Š5 events. $\mathrm{h}^{-1}$. This lack of necessity of sleepiness probably contributed to the mean MSLT SOL of 9.7 min in this study, compared with a mean value of $6 \mathrm{~min}$ in the SAHS patients of RoEHRS et al. [9]. In all other aspects the population of the present study was a typical unselected SAHS population, with a mean AHI of 45 , body mass index (BMI) of 32 and MWT of 27 min, similar to the results of POCETA et al. [14]. It may be that the patients in the present study were less sleepy than in some other studies of sleep apnoeics because these pa-tients had a normal night's sleep at home prior to the testing. Evidence suggests that the lack of patient sleepiness did not have an effect on these results as when a subgroup of patients $(n=75)$ with both an AHI Š 15 and ESS S 11 were examined, they showed similar results to the population as a whole. No significant relationships were seen with AHI, microarousals or desaturation variables and sleepiness, as measured by the ESS, MSLT and MWT.

Alternatively, was the current study population not sleepy as defined by the MSLT, because the daytime testing schedule was busy (table 3), and so increased alertness? Previous studies have successfully performed the MSLT and MWT on the same day $[10,31,38]$. When performed in the study by MARTIN et al. [10], significant changes in both variables were seen with sleep fragmentation. In addition, HoRne and MinaRd [39] found that a behaviourally active day in normals caused an increase in subjective sleepiness compared to a monotonous laboratory based day. In agreement, KRIBBS et al. [40] compared 10 min nap opportunities before and after a vigilance task. Nap latency decreased after the brief performance task, suggesting that the task potentiated sleepiness rather than promoting alertness. Neither of these studies $[39,40]$ explain the relatively nonsleepy patient population in the present study. Furthermore, most patients usually have an active daytime schedule and this protocol more closely resembles their normal day than merely sitting around or resting in bed trying to fall asleep, the standard day for an MSLT as classically performed. 
The lack of a close correlation between nocturnal measures and daytime function could reflect that either the nocturnal variables conventionally scored do not influence daytime function, or that there are other unknown factors which determine the daytime variables. Altering the microarousal frequency within individuals does alter their daytime function on the tests that have been used [10], thus these are relevant daytime measures. However, there may be more sophisticated sleep-related variables which would better explain the observed variance, such as spectral EEG analysis or detection of arousals from autonomic variables rather than from the EEG. Equally the respiratory measures used, apnoeas and hypopnoeas, may not be the most sensitive measures and perhaps oesophageal pressure swings or inspiratory flattening should be examined.

Nevertheless, it seems likely that across population analyses will be limited in value due to the large number of other factors, including genetic, educational and environmental, which influence both premorbid and morbid daytime function. Patients may have different thresholds for suffering daytime consequences, regardless of disease severity. Sleepiness is context dependent and although patients perform the MSLT/MWT under identical conditions, they differ in terms of their usual daily routines. For example, some patients nap on a daily basis, whilst others not at all perhaps due to different work environments and commitments. Some patients have learnt to adapt to their disease, possibly in terms of an increased arousal threshold, or by better use of behavioural techniques to stay awake and upgrade performance when required. Such factors are likely to vary between individuals. These between subject variances that naturally exist will mask any possible relationships between polysomnographic variables and daytime function.

This study shows that the brief microarousals seen in sleep disordered breathing do not correlate with sleepiness and correlated poorly with daytime dysfunction. In addition it was found that the maintenance of wakefulness test more closely related to daytime function than the multiple sleep latency test.

Acknowledgements The authors thank the technical, nursing and administrative staff of the Scottish National Sleep Centre for the assistance they provided during the study.

\section{References}

1. Roth T, Harste KM, Zorick F, Conway W. Multiple naps and the evaluation of daytime sleepiness in patients with upper airway sleep apnea. Sleep 1980; 3: 425-439.

2. Whyte KF, Allen MB, Jeffrey A, Gould GA, Douglas NJ. Clinical features of the sleep apnoea/hypopnoea syndrome. Q J Med 1989; 72: 659-666.

3. Greenberg GD, Watson RK, Deptula D. Neuropsychological dysfunction in sleep apnea. Sleep 1987; 10: 254-262.

4. Bédard MA, Montplaisir J, Richer F, Malo J. Nocturnal hypoxemia as a determinant of vigilance impairment in sleep apnea syndrome. Chest 1991; 100: 367-370.

5. Cheshire K, Engleman HM, Deary I, Shapir C, Douglas NJ. Factors impairing daytime performance in patients with the sleep apnoea/hypopnoea syndrome. Arch Int Med 1992; 52: 538-541.
6. George CF, Nickerson PK, Hanly PJ, Millar TW, Kryger MH. Sleep apnoea patients have more automobile accidents. Lancet 1987; ii: 447.

7. Kales A, Caldwell AB, Cadieux RJ, Vela-Bueno A, Ruch LG, Mayes SD. Severe obstructive sleep apnea - II: associated psychopathology and psychosocial consequences. J Chronic Dis 1985; 38: 427-434.

8. Guilleminault C, Partinen M, Quera-Salva MA, Hayes B, Dement WC, Nino-Murciia G. Determinants of daytime sleepiness in obstructive sleep apnea. Chest 1988; 94: 3237.

9. Roehrs T, Zorick F, Wittig R, Conway W, Roth T. Predictors of objective level of daytime sleepiness in patients with sleep-related breathing disorders. Chest 1989; 95 : 1202-1206.

10. Martin SE, Engleman HM, Deary IJ, Douglas NJ. The effect of sleep fragmentation on daytime function. Am J Respir Crit Care Med 1996; 153: 1328-1332.

11. Philip P, Stoohs R, Guilleminault C. Sleep fragmentation in normals: a model for sleepiness associated with upper airway resistance syndrome. Sleep 1994; 17: 242-247.

12. Roehrs T, Merlotti L, Petrucelli N, Stepanski E, Roth T. Experimental sleep fragmentation. Sleep 1994; 17: 438443.

13. Carskadon MA, Dement WC, Mitler MM, Roth T, Westbrook PR, Keenan S. Guidelines for the multiple sleep latency test (MSLT): a standard measure of sleepiness. Sleep 1986; 9: 519-524.

14. Poceta JS, Timms RM, Jeong D, Ho S, Erman MK, Mitler MM. Maintenance of wakefulness test in obstructive sleep apnea syndrome. Chest 1992; 101: 893-897.

15. Douglas NJ, Thomas S, Jan MA. Clinical value of polysomnography. Lancet 1992; 339: 347-350.

16. Rechtshaffen A, Kales A. A Manual of Standardised Terminology, Techniques, and Scoring System for Sleep Stages of Human Subjects. Los Angeles, CA, UCLA, 1968.

17. Gould GA, Whyte KF, Rhind GB, et al. The sleep hypopnoea syndrome. Am Rev Respir Dis 1988; 137: 895-898.

18. Martin SE, Engleman HM, Kingshott RN, Douglas NJ. Microarousals in patients with sleep apnoea/hypopnoea syndrome. J Sleep Res 1997; 6: 276-280.

19. Engleman HM, Martin SE, Deary IJ, Douglas NJ. Effect of continuous positive airway pressure treatment on daytime function in sleep apnoea/hypopnoea syndrome. Lancet 1994; 343: 572-575.

20. Johns MW. Daytime sleepiness, snoring and obstructive sleep apnea. Chest 1993; 103: 30-36.

21. Zigmond AS, Snaith RP. The hospital anxiety and depression scale. Acta Psychiatr Scand 1983; 67: 361-370.

22. Hunt SM, McEwan J, McKenna SP. Perceived health; age and sex comparisons in a community. $J$ Epidemiol Commun Health 1984; 38: 156-160.

23. Smith IE, Shneerson JM. Is the SF-36 sensitive to sleep disruption? A study in subjects with sleep apnoea. J Sleep Res 1995; 4: 183-188.

24. Gronwall D. Paced Auditory Serial Addition Test: a measure of recovery from concussion. Percept Mot Skills 1977; 44: 367-373.

25. Lezak MD. Neuropsychological Assessment, 2nd ed. New York, Oxford University Press, 1983.

26. Thorpy MJ. The clinical use of the multiple sleep latency test. Sleep 1992; 15: 268-276.

27. Engleman HM, Martin SE, Deary 1J, Douglas NJ. Effect of CPAP therapy on daytime function in patients with mild sleep apnoea/hypopnoea syndrome. Thorax 1997; 52: $114-119$. 
28. Grant I, Heaton RK, McSweeney J, Adams KM, Timms RM. Neuropsychologic findings in hypoxemic chronic obstructive pulmonary disease. Arch Intern Med 1982; 142: 1470-1476.

29. Kingshott RN, Sime PJ, Engleman HM, Douglas NJ. Self assessment of daytime sleepiness: patient versus partner. Thorax 1995; 50: 994-995.

30. Kim HC, Young T, Matthews CG, Weber SM, Woodard AR, Palta M. Sleep-disordered breathing and neuropsychological deficits: a population-based study. Am J Respir Crit Care Med 1997; 156: 1813-1819.

31. Sangal RB, Thomas L, Mitler MM. Maintenance of wakefulness test and multiple sleep latency test. Measurement of different abilities in patients with sleep disorders. Chest 1992; 101: 898-902.

32. Collard P, Dury M, Delguste P, Aubert G, Rodenstein D. Movement arousals and sleep-related disordered breathing in adults. Am J Respir Crit Care Med 1996; 154: 454-459.

33. Whyte KF, Allen MB, Fitzpatrick MF, Douglas NJ. Accuracy and significance of scoring hypopnoeas. Sleep 1992; 15: 257-260.

34. Harrison Y, Bright V, Home JA. Can normal subjects be motivated to fall asleep faster? Physiol Behav 1996; 60: 681-684.

35. Roehrs T, Roth T. Multiple sleep latency test: technical aspects and normal values. J Clin Neurophysiol 1992; 9: 63-67.

36. Bennett LS, Stradling JR, Davies RJO. A behavioural test to assess daytime sleepiness in obstructive sleep apnoea. J Sleep Res 1997; 6: 142-145.

37. Engleman HM, Hirst WSJ, Douglas NJ. Under-reporting of sleepiness and driving impairment in patients with the sleep apnoea/hypopnoea syndrome. J Sleep Res 1997; 6: 272-275.

38. Sangal RB, Thomas L, Psg T, Mitler MM. Disorders of excessive sleepiness: treatment improves ability to stay awake but does not reduce sleepiness. Chest 1992; 102: 699-703.

39. Home JA, Minard A. Sleep and sleepiness following a behaviourally 'active' day. Ergonomics 1985; 28: $567-$ 575.

40. Kribbs NB, Packe AI, Dinges DF. Modification of physiological sleep tendency with a prior performance task. Sleep Res 1994; 23: 129. 